\title{
Social and health policies or interventions to tackle health inequalities in European cities: a scoping review
}

Mariona Pons-Vigués ${ }^{1,2,3^{*}}$, Èlia Diez ${ }^{1,2,4}$, Joana Morrison ${ }^{5,2,1,4}$, Sergio Salas-Nicás ${ }^{1}$, Rasmus Hoffmann ${ }^{6}$, Bo Burstrom7, Jitse P van Dijk $k^{8,9}$ and Carme Borrell ${ }^{1,2,4,10}$

\begin{abstract}
Background: Health inequalities can be tackled with appropriate health and social policies, involving all community groups and governments, from local to global. The objective of this study was to carry out a scoping review on social and health policies or interventions to tackle health inequalities in European cities published in scientific journals.

Methods: Scoping review. The search was done in "PubMed" and the "Sociological Abstracts" database and was limited to articles published between 1995 and 2011. The inclusion criteria were: interventions had to take place in European cities and they had to state the reduction of health inequalities among their objectives.

Results: A total of 54 papers were included, of which $35.2 \%$ used an experimental design, and $74.1 \%$ were carried out in the United Kingdom. The whole city was the setting in $27.8 \%$ of them and $44.4 \%$ were based on promoting healthy behaviours. Adults and children were the most frequent target population and half of the interventions had a universal approach and the other half a selective one. Half of the interventions were evaluated and showed positive results.
\end{abstract}

Conclusions: Although health behaviours are not the main determinants of health inequalities, the majority of the selected documents were based on evaluations of interventions focusing on them.

Keywords: Health inequalities, Public policies, Interventions, Cities, Urban health, Scoping review

\section{Background}

Disadvantaged populations have worse health and higher morbidity and mortality [1]. These inequalities are due to different opportunities and resources in relation to health according to social class, gender, country of origin, territory and age $[2,3]$. Health inequalities tend to be greater in urban areas with disadvantaged and poor populations, affecting, as a result, all city residents [4]. Although cities offer many opportunities, jobs and services, their density, diversity, urban segregation and heterogeneous socioeconomic characteristics contribute to inequalities in health [5].

\footnotetext{
* Correspondence: mponsv@idiapjgol.info

'Agència de Salut Pública de Barcelona, Barcelona, Spain

${ }^{2}$ CIBER Epidemiología y Salud Pública (CIBERESP), Madrid, Spain

Full list of author information is available at the end of the article
}

Research on the association between place or environment and health and well-being has been carried out by many scholars from a diversity of disciplines focusing on assessing differences between and within cities [6]. This research has permitted identification of the determinants of health inequalities in urban areas or neighbourhoods which are: the physical context (e.g. environmental characteristics, etc.) including the built-up environment (e.g. urbanisation and housing) and the socioeconomic context (e.g. economic factors, employment, public services, safety, etc.) (see Figure 1). Furthermore, the way in which cities are organised and managed has an impact on health inequalities, and can contribute to exacerbate or reduce them [7-9]. Health in urban populations is in turn shaped by municipal determinants and global and national trends [6]. Cities are complex systems, and, in 


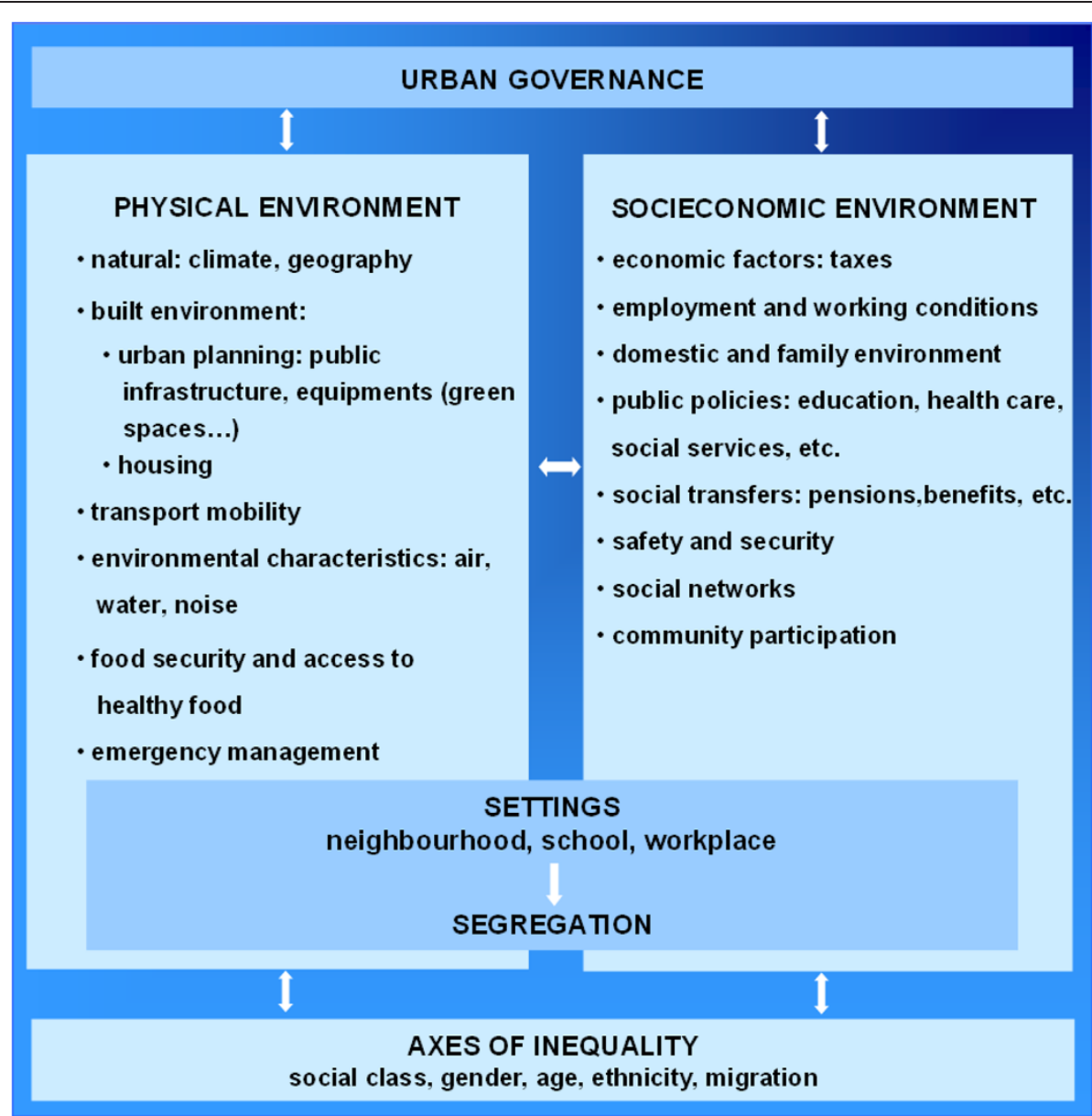

Figure 1 The conceptual framework for the social determinants of health inequalities in cities of Europe [8].

consequence, urban health depends on many interactions [7].

Health inequalities can be tackled with appropriate health and social policies cutting across sectors, involving all community groups and governments, from local to global [4]. Governance has been defined as the relevant process by which governments and other social organizations and actors interact with citizens and make decisions in a complex and globalized world [10]. Effective multilevel governance is clearly cross-sectoral and participative. Despite the diversity of countries' regulations, local governments have the power to face health inequalities. Municipal sectors such as urban planning, culture, leisure, education, environment, health services, social services, housing, etc. have a clear impact on the health of the citizens [7-9].

Socioeconomic inequalities in health have been recognized at least since the 1980s as being an important public health issue [11]. Europe was the only World Health Organization (WHO) Region to develop its own targets for the Health For All (HFA) strategy in 1985, with
Target 1 of the 38 Targets focusing on the reduction of inequalities in health $[9,12]$.

However, only $1.7 \%$ of the documents published in the PubMed database related to Epidemiology or Public Health mention inequalities or determinants of health in the title or abstract [13]. The majority of these health inequalities studies have been conducted to describe and analyse inequalities and are not focused on policies or interventions to reduce them in urban areas [14-17].

Although some countries are increasingly working to achieve health equity, there is little literature describing effective experiences and even less at the urban level. Given that the majority of the world population lives in cities, where health inequities are increasing [4] and that local governments have widely ranging capacities to intervene upon them, an updated review on published interventions to reduce health inequalities in cities may be helpful for future related actions. Therefore, the objective of this study was to carry out a scoping review on social and health policies or interventions to tackle health inequalities in European cities published in scientific journals. 
The potential research questions revolve around what is published, how this evolves, and what types of studies predominate in European cities. For this reason, evaluating the effectiveness of interventions was not the principal aim of this scoping review.

This study forms a part of the Ineq-Cities project: Socioeconomic inequalities in mortality - evidence and policies in cities of Europe (funded by DG-SANCO of the European Union); for that reason, the review focused on European cities. Hence, the project aims to study socioeconomic inequalities in mortality within European cities, as well to identify social and health policies and interventions implemented to address them.

\section{Methods}

\section{Data sources}

A scoping review $[18,19]$ was carried out to select papers on policies or interventions to tackle inequalities in health in European urban areas published in scientific journals. We sought primarily to perform a mapping of the published papers; therefore, the main objective was not to assess the quality. Consequently, we opted for a scoping review which has been described as a process of mapping the existing literature or evidence base, exploring it without describing findings in detail $[18,19]$. In this complex topic, a scoping review is an important and necessary first step before undertaking a more intensive knowledge synthesis because it helps identify appropriate parameters and potential scope [19].

A bibliographic search was done in the National Library of Medicine's PubMed database and in the CSA
Sociological Abstracts database. Biomedical and social databases were chosen to cover different fields and disciplines related to the topic. In addition, a manual search in the reference lists of the papers selected through these two electronic databases was performed [20]. These sources were combined in order to capture as much relevant information as possible. Table 1 illustrates the search strategies and the inclusion and exclusion criteria. The search was limited to papers related to European cities and published between January 1995 and March 2011. Additional requirements for papers were: 1) presentation of the execution and/or results directly related to interventions or policies to reduce health inequalities (interventions had to state the reduction of health inequalities among their objectives), 2) written in English, Spanish or French, and 3) published in peer-reviewed journals. Theoretical papers and those only mentioning health inequalities as a conclusion or recommendation were excluded. As the research questions were what is published, how this evolves and what types of studies predominate, papers were not excluded due to the methodology used.

\section{Study selection}

Figure 2 represents the data extraction flow chart. The searches in Pubmed and Sociological Abstracts were carried out on the 1st of April 2011, and identified 1162 papers (849 papers from Pubmed and 313 from Sociological Abstracts). All these citations were systematically screened and evaluated by one author to exclude publications irrelevant to the inclusion criteria. Therefore, according the

Table 1 Search strategy applied in Pubmed and Sociological Abstracts databases

\begin{tabular}{|c|c|}
\hline \multirow[t]{5}{*}{ INCLUSION criteria } & European Cities \\
\hline & Papers published between January 1995-March 2011 \\
\hline & Papers published in peer-reviewed journals \\
\hline & Papers on policies or interventions to reduce health inequalities \\
\hline & Papers in English, Spanish or French \\
\hline \multirow[t]{2}{*}{ EXCLUSION criteria } & Theoretical papers on policies or interventions to reduce health inequalities \\
\hline & Papers that only talk about reducing inequalities in the conclusions or recommendations \\
\hline Pubmed search & $\begin{array}{l}\text { (health equit* [Title/Abstract] OR health inequalit* [Title/Abstract] OR health inequit* [Title/Abstract] OR disparit* } \\
\text { [Title/Abstract] OR depriv* [Title/Abstract] OR social justice* [Mesh]) AND (policy [Mesh] OR policy making [Mesh] OR } \\
\text { health policy [Mesh] OR organizational policy [Mesh] OR health plan implementation [Mesh] OR community health } \\
\text { planning [Mesh] OR government program [Mesh] OR program evaluation [Mesh] OR intervention* [Title/Abstract] OR } \\
\text { policy* [Title/Abstract] OR policies [Title/Abstract] OR plan [Title/Abstract] OR action [Title/Abstract]) AND (Urban } \\
\text { renewal [Mesh] OR Urban Health [Mesh] OR urban regeneration [Title/Abstract] OR Cities [Mesh] OR city planning } \\
\text { [Mesh] OR urban area [Title/Abstract] OR city* [Title/Abstract] OR cities [Title/Abstract] OR healthy city* [Title/Abstract] } \\
\text { OR municipal* [Title/Abstract] OR town [Title/Abstract]) }\end{array}$ \\
\hline $\begin{array}{l}\text { CSA Sociological abstracts } \\
\text { search }\end{array}$ & 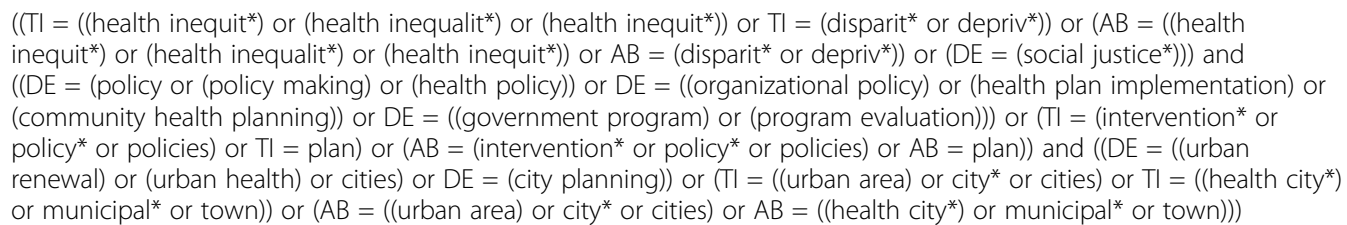 \\
\hline
\end{tabular}




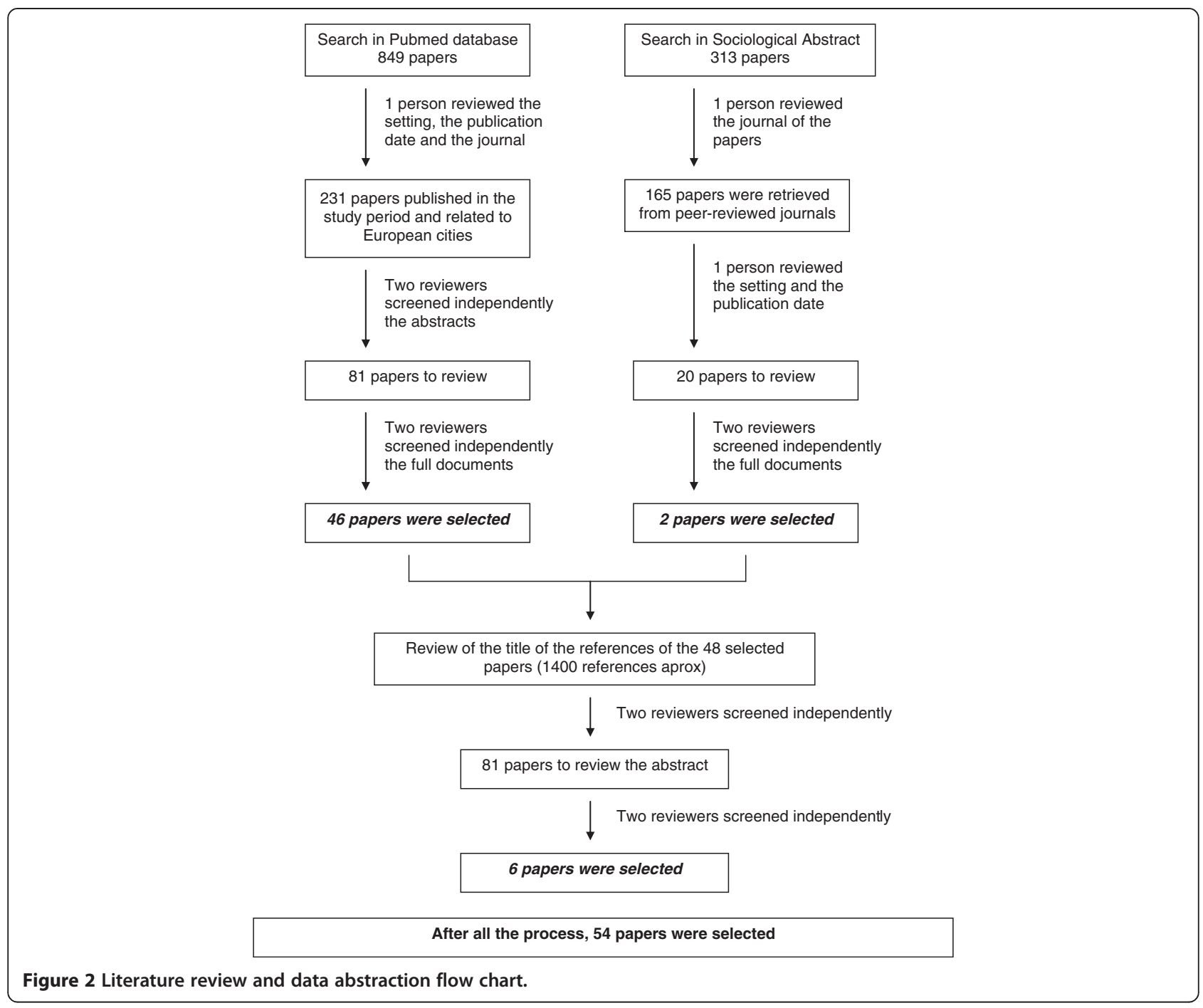

Pubmed search, 231 papers related to European cities had been published in the study period. Two reviewers independently screened these 231 abstracts, and 81 papers were selected to be completely reviewed. Finally, 46 of these papers were included in the study.

With reference to the Sociological Abstracts search, 165 papers were retrieved from peer-reviewed journals. Nevertheless, only 20 of them were related to European cities and published in the study period. Two reviewers independently screened these 20 papers and two of them were included in the study. In both searches, the disagreement between the reviewers about 22 documents was resolved by a third reviewer.

The titles of the documents referenced in the $48 \mathrm{pa}-$ pers selected were screened to exclude obviously irrelevant or duplicate documents. After that, their abstracts were screened independently by two reviewers (MP, JM) who had previously screened the papers in order to find additional ones to be included. The same criteria formerly used were applied. This manual search in the selected papers' reference lists led to the identification of 6 new papers. In consequence, the entire review process resulted in the selection of 54 papers.

\section{Data extraction, variables and data analysis}

The following data were extracted from each publication: authors, year of publication, goal of the paper, study design, city and year of the intervention, target population of the intervention, intervention description, evaluation of the intervention and results or health outcomes of the paper. This information for each paper was captured in a table displayed in the annex of this review (see Additional file 1: Tables S1-S5), and in a database. Five researchers participated independently in the study selection and the data extraction. 
The main characteristics of the documents were classified and are summarized in Tables 2 and 3, taking into account the following variables:

- Type of intervention to reduce health inequalities: 1) Promotion of health behaviours, 2) Healthy settings (where people actively use and shape the environment; as for example, school, workplace, neighbourhood and community), 3) Socioeconomic context (economic factors, employment and working conditions, public services and social transfers), 4) Physical context (climate, built-up environment and environmental characteristics) and 5) Combined approach. This taxonomy was based on the IneqCities project conceptual framework [8,21], (Figure 1), adapted from the one proposed by the WHO Hidden Cities Report [4] and from Vlahov [5].

- Study design of the paper: documents were classified according to the design type of the research conducted. The categories were: experimental studies, quasi-experimental studies, observational studies, qualitative studies, reviews, health impact assessment studies and mixed-methods (studies combining two or more designs).

- Country of city intervention: this variable described the cities where interventions were developed according to the home country. An extra category was added when the intervention took place simultaneously in different cities in different European countries.

- Setting of the intervention: this variable indicates where the intervention was performed and it was categorised as: the entire city, a neighbourhood or area, a healthcare or social center, school, or a specific community group.

- Target population of the intervention: a variable consisting of three categories according to the age of the participants: children, adult and all ages.

- Focus of the intervention: a dichotomous variable indicating whether the intervention was universal or selective (intervention was addressed to a part of the population with some risk or vulnerable features).

- Evaluation of the intervention: this variable collects information from the paper about whether the intervention had been evaluated, and the type of result (positive, negative or partially positivenegative). Some interventions could have been evaluated but if this had not been indicated in the paper it was not stated. It includes different types of evaluation: efficiency, effectiveness, impact, monitoring, satisfaction, etc.

- Year of publication categorized in three groups: 1995-2000, 2001-2005 and 2006-2011.

\section{Results}

The entire review process led to the selection of 54 papers. The main characteristics are presented in Table 2 and 3 summarizes the objective according to the type of intervention, the setting and the target population.

Although publication of interventions tackling health inequalities has increased considerably over the last 15 years, the number of documents is still very small. Between 1995 and 2000, six papers were published, whereas in the past 6 years (2006 - April 2011), 26 papers had been published (48.2\% of the documents of this review). Nineteen of the papers reviewed (35.2\%) used an experimental design such as randomised controlled trials, followed by 13 quasiexperimental studies (24.1\%). $16.7 \%$ of the documents (9 papers) were observational studies whereas 9.2\% were review (5 papers) and qualitative studies (5 papers).

Forty of the 54 studies $(74.1 \%)$ were carried out in cities of the United Kingdom. The 18 remaining papers were divided between cities in Spain (5 studies, 9.2\%), the Netherlands (4 studies, 7.4\%), Germany (2 studies, $3.7 \%$ ), France (1 study, 1.9\%) and other European countries (2 multicentric studies, 3.7\%). The setting of the intervention is more evenly distributed. The setting of 15 papers was the whole city (27.8\%), in 13 it was a deprived neighbourhood (24.1\%) and in 12 a healthcare or social center (22.2\%).

The majority of the interventions targeted both adult and child populations (23 studies, $42.6 \%$ ). Half of them used a universal approach, and the other half a selective one. The majority of the interventions were evaluated (46 interventions, 79.3\%) and 29 of them had positive results $(53.7 \%)$. The rest of the evaluation showed that 8 interventions (14.8\%) were not effective, 7 (13.0\%) showed both positive results and negative results, and 2 interventions $(3.7 \%)$ did not mention the results of their evaluation. Not all of the evaluated interventions used health indicators as outcome measures. Further details of the evaluation of each intervention or policy are available in the supplementary data.

Almost half of the interventions promoted healthy behaviours (24 documents, 44.5\%), for example to promote oral health programmes, increase physical activity, decrease cardiovascular risk factors, etc.; 10 interventions (18.5\%) promoted healthy settings, for example to improve road safety, redesign a playground, etc.; 6 interventions $(11.1 \%)$ targeted the socioeconomic context, for example: transport policy, food services, etc.; 4 interventions were focused on the physical context (7.4\%), for example: neighbourhood renewal, etc.; and 10 interventions (18.5\%) used a combined approach, for example: environmental and educational interventions to promote water consumption. Further details of the documents employing these five approaches are available in the supplementary data. 
Table 2 Descriptive characteristics of the 54 papers obtained classified by type of intervention

\begin{tabular}{|c|c|c|c|c|c|c|c|}
\hline & \multicolumn{5}{|c|}{ Type of intervention } & \multicolumn{2}{|c|}{ Total } \\
\hline & $\begin{array}{l}\text { Promote healthy } \\
\text { behaviours } \\
\text { (N = } 24-44.5 \%)\end{array}$ & $\begin{array}{l}\text { Promote healthy } \\
\text { settings } \\
(\mathrm{N}=10-18.5 \%)\end{array}$ & $\begin{array}{l}\text { Socio-economic } \\
\text { context } \\
(\mathrm{N}=6-11.1 \%)\end{array}$ & $\begin{array}{l}\text { Physical } \\
\text { context } \\
(\mathrm{N}=4-7.4 \%)\end{array}$ & $\begin{array}{l}\text { Combined } \\
\text { approach } \\
(\mathrm{N}=10-18.5 \%)\end{array}$ & $\mathbf{N}$ & $\%$ \\
\hline \multicolumn{8}{|l|}{ Study design } \\
\hline Experimental & 11 & 3 & 2 & 0 & 3 & 19 & 35.2 \\
\hline Quasi-experimental & 5 & 5 & 0 & 1 & 2 & 13 & 24.1 \\
\hline Observational & 5 & 1 & 2 & 1 & 0 & 9 & 16.7 \\
\hline Qualitative & 1 & 1 & 0 & 0 & 3 & 5 & 9.2 \\
\hline Review & 1 & 0 & 0 & 2 & 2 & 5 & 9.2 \\
\hline Health impact assessment & 0 & 0 & 2 & 0 & 0 & 2 & 3.7 \\
\hline Mixed methods & 1 & 0 & 0 & 0 & 0 & 1 & 1.9 \\
\hline \multicolumn{8}{|l|}{ Country } \\
\hline United Kingdom & 19 & 9 & 4 & 4 & 4 & 40 & 74.1 \\
\hline Spain & 0 & 0 & 2 & 0 & 3 & 5 & 9.2 \\
\hline Netherlands & 4 & 0 & 0 & 0 & 0 & 4 & 7.4 \\
\hline Germany & 0 & 0 & 0 & 0 & 2 & 2 & 3.7 \\
\hline France & 1 & 0 & 0 & 0 & 0 & 1 & 1.9 \\
\hline Different European countries & 0 & 1 & 0 & 0 & 1 & 2 & 3.7 \\
\hline \multicolumn{8}{|l|}{ Setting } \\
\hline City & 3 & 2 & 4 & 3 & 3 & 15 & 27.8 \\
\hline Neighbourhood & 6 & 3 & 1 & 1 & 2 & 13 & 24.1 \\
\hline Healthcare center & 11 & 0 & 0 & 0 & 1 & 12 & 22.2 \\
\hline School & 2 & 4 & 1 & 0 & 2 & 9 & 16.7 \\
\hline Community group & 2 & 1 & 0 & 0 & 2 & 5 & 9.2 \\
\hline \multicolumn{8}{|l|}{ Target population } \\
\hline Adults & 14 & 1 & 1 & 0 & 2 & 18 & 33.3 \\
\hline Children & 4 & 6 & 0 & 1 & 2 & 13 & 24.1 \\
\hline Adults and Children & 6 & 3 & 5 & 3 & 6 & 23 & 42.6 \\
\hline \multicolumn{8}{|l|}{ Focus } \\
\hline Universal & 10 & 6 & 3 & 3 & 5 & 27 & 50.0 \\
\hline Selective & 14 & 4 & 3 & 1 & 5 & 27 & 50.0 \\
\hline \multicolumn{8}{|l|}{ Evaluation of the intervention } \\
\hline Yes, positive result & 13 & 6 & 3 & 1 & 6 & 29 & 53.7 \\
\hline Yes, negative result & 5 & 1 & 0 & 0 & 2 & 8 & 14.8 \\
\hline Yes, positive and negative results & 2 & 1 & 1 & 3 & 0 & 7 & 13.0 \\
\hline Yes, missing result & 0 & 2 & 0 & 0 & 0 & 2 & 3.7 \\
\hline No & 1 & 0 & 1 & 0 & 0 & 2 & 3.7 \\
\hline Missing & 3 & 0 & 1 & 0 & 2 & 6 & 11.1 \\
\hline \multicolumn{8}{|l|}{ Year of publication } \\
\hline 2006-2011 & 14 & 6 & 2 & 0 & 4 & 26 & 48.2 \\
\hline 2001-2005 & 7 & 1 & 4 & 4 & 4 & 20 & 37.0 \\
\hline 1995-2000 & 3 & 3 & 0 & 0 & 2 & 8 & 14.8 \\
\hline
\end{tabular}


Table 3 Interventions classified according to type of intervention, setting and target population

\begin{tabular}{|c|c|c|}
\hline $\begin{array}{l}\text { Type of } \\
\text { intervention }\end{array}$ & Setting & Adult \\
\hline \multirow{11}{*}{$\begin{array}{l}\text { Promote } \\
\text { healthy } \\
\text { behaviours }\end{array}$} & \multirow[t]{2}{*}{ City } & Physical and cognitive training programme \\
\hline & & Different activities to promote healthy eating \\
\hline & \multirow{2}{*}{$\begin{array}{l}\text { Neighbourhood } \\
\text { or area }\end{array}$} & A weight management programme \\
\hline & & Physical exercise program \\
\hline & \multirow[t]{7}{*}{$\begin{array}{l}\text { Healthcare or } \\
\text { social center }\end{array}$} & $\begin{array}{l}\text { A web-based intervention to support self- } \\
\text { management in patients with coronary heart } \\
\text { disease }\end{array}$ \\
\hline & & Assessing CVD risk in pharmacies \\
\hline & & Preventive care of patients with high CV risk \\
\hline & & Cultural approach to CV risk prevention program \\
\hline & & $\begin{array}{l}\text { Behavioural counselling on fruit and vegetables } \\
\text { consumption }\end{array}$ \\
\hline & & Health shop in community centre \\
\hline & & Cognitive Behaviour Theraphy for smokers \\
\hline
\end{tabular}

School

Community

group

City

healthy

settings

Socioeconomic City context

Healthcare or social center

School

\section{Community} group

Target population

Children

Adult \& Children

Health check tests and a poster campaign

Reduce teenage pregnancy Introduction of health brokers

Dietary health education program

to promote health

Oral health promotion program

Intensive home-visiting program

Interventions to prevent mother-to-child transmission of HIV

Introduction of asthma specialist nurses

Individual asthma education programme
Teacher-supervised toothbrushing

Intervention for diabetes support and education

Health education and physical exercise programme
Domestic water

fluoridation

Receiving pasteurised cows' milk by 6 months of age

Road safety program

Free healthy school meals

Playground redesign intervention
Free smoke alarms and fire safety information

Improved physical access to high-quality 'healthy' foods

Installation of central heating
Primary care reform

Transport strategy

Early Years Centers provide high quality day care
Neighbourhood

or area

Healthcare or social center

School

Community

group 
Table 3 Interventions classified according to type of intervention, setting and target population (Continued)

\begin{tabular}{|c|c|c|c|c|}
\hline \multirow{6}{*}{$\begin{array}{l}\text { Physical } \\
\text { context }\end{array}$} & \multirow[t]{2}{*}{ City } & & Traffic calming & Urban regeneration programs \\
\hline & & & & $\begin{array}{l}\text { Interventions to improve } \\
\text { housing }\end{array}$ \\
\hline & \multicolumn{2}{|l|}{$\begin{array}{l}\text { Neighbourhood } \\
\text { or area }\end{array}$} & & Neighbourhood renewal \\
\hline & \multicolumn{2}{|l|}{$\begin{array}{l}\text { Healthcare or } \\
\text { social center }\end{array}$} & & \\
\hline & \multicolumn{2}{|l|}{ School } & & \\
\hline & \multicolumn{2}{|l|}{$\begin{array}{l}\text { Community } \\
\text { group }\end{array}$} & & \\
\hline \multirow{8}{*}{$\begin{array}{l}\text { Combined } \\
\text { approach }\end{array}$} & \multirow[t]{2}{*}{ City } & & & Healthy cities \\
\hline & & & & $\begin{array}{l}\text { Strategies to reduce health } \\
\text { inequalities }\end{array}$ \\
\hline & \multirow{2}{*}{\multicolumn{2}{|c|}{$\begin{array}{l}\text { Neighbourhood } \\
\text { or area }\end{array}$}} & & Family Support Services \\
\hline & & & & $\begin{array}{l}\text { Social and health maternal and } \\
\text { child intervention }\end{array}$ \\
\hline & \multicolumn{2}{|l|}{$\begin{array}{l}\text { Healthcare or } \\
\text { social center }\end{array}$} & & $\begin{array}{l}\text { Postnatal Support Health } \\
\text { Visitor }\end{array}$ \\
\hline & \multicolumn{2}{|l|}{ School } & $\begin{array}{l}\text { Environmental and } \\
\text { educational intervention }\end{array}$ & \\
\hline & \multirow{2}{*}{$\begin{array}{l}\text { Community } \\
\text { group }\end{array}$} & Race equality policies & & \\
\hline & & $\begin{array}{l}\text { Social care and health follow-up programme } \\
\text { targeting homeless tuberculosis patients }\end{array}$ & & \\
\hline
\end{tabular}

CVD: Cardiovascular diseases.

CV: Cardiovascular.

HIV: Human Immunodeficiency Virus.

More than half of the interventions promoting health behaviour had adults as the target population (14 papers), 14 papers used a selective approach, 13 papers were evaluated and found positive results. Eleven of these 24 interventions were set in healthcare centers. Examples of interventions developed in health centres and aimed at the adult population are behavioural counselling on fruit and vegetables consumption [22], and setting up a health shop in a community centre [23].

Six of the 10 interventions promoting healthy settings targeted children, had a universal approach, were evaluated and showed positive results. Four of these interventions were developed at schools, for example, playground redesign interventions [24,25].

Five of the 6 papers about interventions related to socioeconomic context and material resources were addressed to the whole population, three papers took a universal approach, four papers addressed the whole city and 4 papers were evaluated and found positive results. Primary health care reform [26] was an example of intervention aimed at the entire population of the city.

The physical context was the focus of only 4 interventions. Three of them targeted the whole population, were evaluated and obtained both positive and negative results.
More than half of the interventions using a combination approach targeted the whole population (6 papers), used a universal approach (5 papers), were evaluated and obtained positive results (6 papers). Three of these interventions addressed the whole city, for example interventions related to the healthy cities strategy [27].

During the review process some protocols of design, implementation or interventions evaluation were identified. Although they were out of the scope of this study, we considered that they provided interesting information. For that reason, these 4 studies are cited in this paper [28-31].

\section{Discussion}

This project identified 54 papers published in scientific journals related to social and health policies or interventions to tackle health inequalities in European cities. A third of the studies used an experimental design, most of them were carried out in the United Kingdom, a quarter had either the whole city as the setting or a deprived neighbourhood and half of them promoted healthy behaviours. The majority of the interventions targeted both adults and children, half of them used a universal approach and the other half a selective one. Half of the interventions were evaluated and had positive results. 
Although the volume of publications in this field has increased over the years, there are still relatively few papers published in scientific journals related to policies and interventions focussed on real experiences to tackle health inequalities. Different factors may contribute to this fact. As the literature shows, searching for studies on the social determinants of health or health inequalities is difficult and time-consuming [16]. Although the combination of heterogeneous sources of data were included in order to include as much relevant information as possible, there are probably many documents describing interventions to reduce social inequalities in health which have not been collected in this review because they are in the grey literature (for example, European Portal for Action on Health Inequalities [32]) or because there is no evidence of this purpose in the objective or in the summary of the paper. This has already been identified as a real gap in a review paper on the wider social determinants of health [16]. As we had a more specific aim, it is probable that there is even less available scientific literature. Although some useful interventions have been published in grey literature and reports or in presentations [12], it would be interesting to publish them in scientific journals as this is the best way to reach scholars and improve evidence based scientific knowledge.

In addition, we were looking for policies and interventions at the local level. The reduction of health inequalities cannot be done only with policies undertaken in urban areas; there are many health determinants which are the responsibility of the national or regional government $[3,15]$. Some important universal policies with great potential effect on health inequalities, implemented on a national level, may therefore be overlooked (e.g. maternal and child health services). However, local governments have certain competences in a number of sectors from which they can contribute in the reduction of socio-economic health inequalities [33]. As cities are an important setting for intervention governance, it is necessary to show what is being done at this level. In the health policy documents of some cities or counties, it is possible to see that certain strategies are planned [34], but often these strategies do not end up being described to the scientific community. This may be due to the heavy workload of people responsible for such interventions, but also because it is very difficult to publish them as local experiences are not as attractive to scientific journals as national or international strategies. As social health inequalities are an important public health issue, it might be interesting to promote publishing of strategies carried out, or at least to increase the number of published interventions in scientific journals. Health inequalities are present in all countries; therefore, the experience of interventions carried out in other cities to tackle them might prove to be very interesting and helpful.
In children and in adults, interventions are mainly focussed on the lifestyle drift and on promoting healthy behaviours [35]. As Bambra [36] reported, policy has had a tendency to focus on healthy behaviour interventions rather than tackling upstream, distal causes such as poor living conditions. This approach contrasts with evidence which suggests that health inequalities tend to persist between socioeconomic groups even if lifestyle factors are equalized [36]. This poor progress is probably attributable to the fact that studying the impact on health inequalities of interventions in upstream structural determinants is more difficult due to the complex pathways between these determinants and health inequalities. However, the recent WHO review of social determinants suggests that addressing the "causes of the causes" is the right way to proceed on these, ensuring that people have the skills and control over their lives to be able to change behaviour [37].

Although many cities are making efforts to tackle inequalities in health [12], it is necessary to move from a traditional health education focus on lifestyle determinants to more strategic interventions on structural determinants (as many of those presented in Figure 1). The challenge of reducing health inequalities requires policies and interventions in all sectors of society, because their causes are complex $[6,7,9]$. That is to say that health inequities challenge the traditional division of societies and their governments into sectors for organizational purposes [35]. Many policies aiming to address social determinants require intersectoral action [38] and community participation, two principles of action proposed by the Commission on Social Determinants of Health [15]. This is especially relevant in cities, according to statements by Healthy Cities [39]. A recent paper suggests that intersectoral approaches to health equity are feasible in a variety of social, economic and political systems [38]. In consequence, the intersectoral approach is essential to improve the health, wellbeing and quality of life of citizens. All community sectors are essential in addressing health policy [40]. Unfortunately, these aspects are not too clear or evident in many of papers in this review. Achieving community participation and working intersectorally is not an easy task, even though it is considered important [40]. Evidence shows that intersectoral action is more effective than tackling health inequalities from the health sector only [41]. A useful tool to promote effective work in this sense is the Urban Health Equity Assesment and Response Tool (Urban Heart), a clear guide for policy makers at local and national levels to address health inequalities in cities [42]. Moreover, intersectoral action facilitates the WHO policy Health in All Policies, which takes into account the impact on health of all policies, such as education, environment, economic policies, housing, or transport mobility [43]. In addition, current experience shows that health impact assessment could play 
an important role in the development of the Health in All Policies strategy. Health impact assessment is an important methodology to support decisions in policy-making [44].

The United Kingdom (UK) is one of the countries with a longer historical background of working on health inequalities $[45,46]$ and, for example, London has a specific strategy to tackle health inequalities [34] which has been supported by different governments. This fact is corroborated in this paper because the UK is the country where most published interventions were found. In addition, English people might have more facilities to publish because of the language. We found more papers in Pubmed than in the Sociological Abstracts database probably because the latter tends to focus on international literature in sociology and disciplines traditionally more related to the social and behavioural sciences rather than to health. However, social and economic factors are being considered more and more as determinants of health inequalities so we consider that this trend may change. In consequence, this issue may deserve further research.

There is still little experience of evaluating the impact of interventions to reduce health inequalities $[27,47,48]$. Most of the evaluations reviewed in this study concerned healthy behaviours and proximal outcomes. Evaluating upstream interventions presents additional methodological challenges. Evidence is especially needed from evaluations of universal polices [35]. That is particularly important to inform the debate on the most efficient and effective use of scarce resources in financial crisis situations, such as the present global one. In addition, it is necessary because it can happen that some interventions may increase inequalities in the population if they are of greater benefit to advantaged (lower-risk) groups than to disadvantaged (higher-risk) groups [49]. Despite the fact that the aim of this scoping review was not to evaluate the effectiveness or quality of the interventions included $[18,19]$, this is a subject which warrants further study. Note that $54 \%$ of the interventions were evaluated and had positive results. However, not all reported results in terms of health indicators. Even in the UK which has such a long history of tackling social determinants of health, evaluation is sorely lacking [50]. About 35\% of the papers used an experimental design to evaluate the intervention. Overall, the evaluation of effects is the aspect most commonly published in scientific journals rather than accounts of real experiences, which are probably explained to other audiences.

\section{Strengths and limitations of the study}

To our knowledge, this is the first scientific review of the social and health policies or interventions to tackle health inequalities in European cities. Previous papers have reviewed health inequalities interventions at the national level or at the urban level but in the American context (USA and Canada) [33,36]. For that reason, this study can contribute to advancing knowledge in the reduction of socioeconomic health inequalities. At the national level, Bambra et al. combined a rapid review with a Delphi distillation, producing a shortlist of evidence-based recommendations. According to these authors, extensive, specific and robust evidence is urgently needed to guide policy and programmes [36]. Besides, Collins and Hayes demonstrated a pervasiveness of 'behavioural' and 'biomedical' perspectives, and a lack of consideration afforded to the roles and responsibilities of municipal governments, among the health inequities scholarly community [33].

The rigorous procedures used have ensured the validity of the data abstraction. However, the review is not exhaustive because we have not been able to identify all relevant evidence and we did not search in the grey literature. We tried to identify all possible papers published in scientific journals by searching in two different databases, one of which is not medical, in order to capture policies or interventions from other sectors. The combination of heterogeneous sources of data adds value to the results. As one of the additional requirements for inclusion was that papers were written in English, Spanish or French, we believe it has brought a significant change in the results since we only had to exclude two documents.

We must also take into account that the fact that these policies and interventions have been published does not necessarily mean they are still functioning or effective. Furthermore, given the heterogeneity of the objectives of the papers it has not been possible to summarize their main results or conclusions. The articles varied with regard to the richness of information included to describe different aspects of these interventions or policies.

\section{Conclusions}

The volume of publications in this field has increased over the years, however, there are still relatively few papers published in scientific journals related to policies and interventions focussed on real experiences to tackle health inequalities. In addition, although health behaviours are not the main determinants of health inequalities [15], the majority of the documents published in scientific journals were based on interventions focusing on them. In order to increase the policy evidence, to support people working in interventions and to contribute to the incorporation of health in all policies [15], examples of local policies and interventions to reduce inequalities should be published in the scientific literature.

\section{Additional file}

Additional file 1: Table S1. Promotion health behaviours interventions. Table S2. Healthy settings interventions. Table S3. Socioeconomic context interventions. Table S4. Physical context interventions. Table S5. Combined approach interventions. List of the 54 papers selected in this study. 


\section{Competing interests}

The authors declare that they have no competing interests.

\section{Authors' contributions}

$M P V, E D, J M$ and $C B$ are the principal investigators responsible for the conception of the project. In addition, MPV, ED, JM and CB were involved in data collection, analysis of the data and writing the manuscript. SSN, $\mathrm{RH}, \mathrm{BB}$ and JPD have made substantial contributions to interpretation of data; have been involved in drafting the manuscript or revising it critically for important intellectual content. All authors participated in the discussion of the analysis, the editing of the drafts and read and agreed on the final version of this manuscript. All authors have participated sufficiently in the work to take public responsibility for appropriate portions of the content. All authors read and approved the final manuscript.

\section{Acknowledgements}

We would like to express our thanks to Edwin Ng for his collaboration in the design of the Sociological Abstracts search strategy, and to all those INEQ-CITIES researchers who contributed ideas included in this paper. This article has been partially funded by the project INEQ-CITIES, "Socioeconomic inequalities in mortality: evidence and policies of cities of Europe"; project funded by the Executive Agency for Health and Consumers (Commission of the European Union), project n²008 1213.

\section{Author details}

${ }^{1}$ Agència de Salut Pública de Barcelona, Barcelona, Spain. ${ }^{2} \mathrm{CIBER}$ Epidemiología y Salud Pública (CIBERESP), Madrid, Spain. ${ }^{3}$ Institut Universitari d'Investigació en Atenció Primària Jordi Gol (IDIAP Jordi Gol), Barcelona, Spain. ${ }^{4}$ Institut d'Investigació Biomèdica (IIB Sant Pau), Barcelona, Spain. ${ }^{5}$ Department of Epidemiology and Public Health, University College London, London, UK. ${ }^{6}$ Erasmus Medical Center, Rotterdam, Netherlands. ${ }^{7}$ Department of Public Health Sciences, Karolinska Institutet, Stockholm, Sweden. ${ }^{8}$ Department of Community \& Occupational Health, University Medical Center Groningen, Groningen, Netherlands. ${ }^{9}$ Graduate School Kosice Institute for Society and Health, Safarik University, Kosice, Slovakia. ${ }^{10}$ Universitat Pompeu Fabra, Barcelona, Spain.

Received: 29 July 2013 Accepted: 4 February 2014 Published: 24 February 2014

\section{References}

1. Benach J: La desigualdad perjudica seriamente la salud. Gac Sanit 1997, 11(6):255-258.

2. Borrell C, Artazcoz L: Las políticas para disminuir desigualdades en salud Gac Sanit 2008, 22(5):465-473.

3. Comisión para reducir las desigualdades sociales en salud en España: Propuesta de políticas e intervenciones para reducir las desigualdades sociales en salud en España. Gac Sanit 2012, 26(2):182-189.

4. WHO/UN-HABITAT: Hidden Cities: Unmasking And Overcoming Health Inequities In Urban Settings. Geneva: World Health Organization; 2010.

5. Vlahov D, Freudenberg N, Proietti F, Ompad D, Quinn A, Nandi V, et al: Urban as a determinant of health. J Urban Health 2007, 84(Suppl 3):16-26.

6. Galea S, Freudenberg N, Vlahov D: Cities and population health. Soc Sci Med 2005, 60(5):1017-1033.

7. Rydin Y, Bleahu A, Davies M, Dávila JD, Friel S, De Grandi G, et al: Shaping cities for health: complexity and the planning of urban environments in the 21st century. Lancet 2012, 379(9831):2079-2108.

8. Borrell C, Pons-Vigués M, Morrison J, Díez E: Factors and processes influencing health inequalities in urban areas. J Epidemiol Community Health 2013, 67(5):389-391.

9. World Health Organization Regional Office for Europe: Addressing The Social Determinants Of Health: The Urban Dimension And The Role Of Local Government. In Edited by Grady M, Goldblatt P. Copenhagen: WHO; 2012.

10. WHO: Closing The Gap: Policy Into Practice On Social Determinants Of Health. In Discussion Paper. Brazil: World Conference on Social Determinants of Health; 2011.

11. Mackenbach JP, Bakker MJ: European network on interventions and policies to reduce inequalities in health. Tackling socioeconomic inequalities in health: analysis of european experiences. Lancet 2003, 362:1409-1414.
12. Ritsatakis A: Equity and the social determinants of health in European cities. J Urban Health 2013, 90(Suppl 1):92-104.

13. Borrell C, Malmusi D: Research on social determinants of health and health inequalities: evidence for health in all policies. Gac Sanit 2010, 24(Suppl 1):101-108.

14. Mackenbach J, Stirbu I, Roskam A, Schaap M, Menvielle G, Leinsalu M, Kunst A: Socioeconomic inequalities in health in 22 European countries. N Engl J Med 2008, 358(23):2468-2481.

15. CSDH: Closing The Gap In A Generation: Health Equity Through Action On The Social Determinants Of Health. In Final Report Of The Commission On Social Determinants Of Health. Geneva: World Health Organization; 2008.

16. Bambra C, Gibson M, Sowden A, Wright K, Whitehead M, Petticrew M: Tackling the wider social determinants of health and health inequalities: evidence from systematic reviews. J Epidemiol Community Health 2010, 64(4):284-291.

17. Dahlgren G, Whitehead M: European Strategies for Tackling Social Inequities In Health. Concepts and Principles for Tackling Social Inequities in Health. Levelling up (part 2). Denmark: World Health Organization: Studies on social and economic determinants of population health $n^{\circ} 3 ; 2006$.

18. Levac D, Colquhoun H, O'Brien KK: Scoping studies: advancing the methodology. Implement Sci 2010, 5:69.

19. Armstrong R, Hall BJ, Doyle J, Waters E: Cochrane update. 'Scoping The scope' of a Cochrane review. J Public Health (Oxf) 2011, 33(1):147-150.

20. Pope C, Mays N, Popay J: Synthesizing Qualitative and Quantitative Health Evidence: A Guide to Methods. United Kingdom: Open University Press; 2007.

21. Borrell $C$, Palència L, Pons-Vigués $M$, Marí-Dell'Olmo M, Gotsens M, Morrison J, Díez E, INEQCITIES group: Commissioned chapter on inequalities in health. In European Review of Social Determinants of Health, Interim Second Report on Social Determinants of Health and the Health Divide in the European Region. WHO Report led by Professor Sir Michael Marmot; 2011.

22. Steptoe A, Perkins-Porras L, McKay C, Rink E, Hilton S, Cappuccio FP: Behavioural counselling to increase consumption of fruit and vegetables in low income adults: randomised trial. BMJ 2003, 326(7394):855.

23. Forbes $\mathrm{A}$ : A community nurse-led project to tackle health inequalities. Br J Community Nurs 2000, 5(12):610-618.

24. Ridgers ND, Stratton G, Fairclough SJ, Twisk JW: Children's physical activity levels during school recess: a quasi-experimental intervention study. Int J Behav Nutr Phys Act 2007, 4:19.

25. Ridgers ND, Stratton G, Fairclough SJ, Twisk JW: Long-term effects of a playground markings and physical structures on children's recess physical activity levels. Prev Med 2007, 44(5):393-397.

26. Daban F, Pasarín MI, Rodríguez-Sanz M, García-Altés A, Villalbí JR, Cano-Serral G, Borrell C: Evaluation of the primary health care reform: preventive practices and inequalities. Aten Primaria 2007, 39(7):339-346.

27. Ritsatakis A: Equity and social determinants of health at a city level. Health Promot Int 2009, 24(Suppl 1):181-190.

28. Davey RC, Cochrane T, Gidlow C, Fairburn J, Smith G: Design of a pragmatic cluster randomised controlled trial: ecological approach to increasing physical activity in an urban community. Contemp Clin Trials 2008, 29(5):774-782.

29. Hind D, Scott EJ, Copeland R, Breckon JD, Crank H, Walters SJ, et al: A randomised controlled trial and cost-effectiveness evaluation of "booster" interventions to sustain increases in physical activity in middle-aged adults in deprived urban neighbourhoods. BMC Public Health 2010, 10:3.

30. Lyons RA, Towner E, Christie N, Kendrick D, Jones SJ, Hayes M, et al: The advocacy in action study a cluster randomized controlled trial to reduce pedestrian injuries in deprived communities. Inj Prev 2008, 14(2):e1.

31. Wall M, Hayes R, Moore D, Petticrew M, Clow A, Schmidt E, et al: Evaluation of community level interventions to address social and structural determinants of health: a cluster randomized controlled trial. BMC Public Health 2009, 9:207.

32. European Portal for Action on Health Inequalities. http://www.healthinequalities.eu.

33. Collins PA, Hayes MV: The role of urban municipal governments in reducing health inequities: a meta-narrative mapping analysis. Int J Equity Health 2010, 9:13.

34. Borrell C, Morrison J, Burstrom B, Pons-Vigués M, Hoffman R, Gandarillas A, et al: Comparison of health policy documents of cities of Europe: are they oriented to reduce inequalities in health? J Public Health Policy 2013, 34(1):100-120. 
35. World Health Organization Regional Office for Europe: How Can The Health Equity Impact of Universal Policies Be Evaluated? Insights Into Approaches And Next Steps. Copenhagen: World Health Organization; 2011. http://www. euro.who.int/_data/assets/pdf_file/0019/155062/E95912.pdf.

36. Bambra C, Joyce KE, Bellis MA, Greatley A, Greengross S, Hughes S, et al: Reducing health inequalities in priority public health conditions: using rapid review to develop proposals for evidence-based policy. J Public Health (Oxf) 2010, 32(4):496-505.

37. World Health Organization Regional Office for Europe: Review of Social Determinants and the Health Divide in the Who European Region: Final Report. In Edited by Marmot M. Copenhagen: World Health Organization; 2013.

38. Shankardass K, Solar O, Murphy K, Greaves L, O'Campo P: A scoping review of intersectoral action for health equity involving governments. Int J Public Health 2012, 57(1):25-33.

39. WHO European Healthy Cities Network and Network of European National Healthy Cities Networks: Governance for Health at the Local Level: People, Citizens and Assets for Health. Copenhagen: World Health Organization; 2012.

40. Marmot Review: Fair Society, Healthy Lives: Strategic Review of Health Inequalities in England Post 2010. England: Marmot Review; 2010. Available in: www.ucl.ac.uk/marmotreview.

41. World Health Organization, Public Health of Agency of Canada: Health Equity through Intersectorial Action: An analysis of 18 country case studies. Canada: World Health Organization; 2009.

42. Kumaresan J, Prasad A, Alwan A, Ishikawa N: Promoting health equity in cities through evidence-based action. J Urban Health 2010, 87(5):727-732.

43. Ståhl T, Wismar M, Ollila O, Lahtinen E, Leppo K (Eds): Health In All Policies: Prospects And Potentials. Finland: Ministry of Social Affairs and Health; 2006.

44. Esnaola S, Bacigalupe A, Sanz E, Aldasoro E, Calderón C, Zuazagoitia J, Cambra K: Health impact assessment: one way to introduce health in all policies. SESPAS Report 2010. Gac Sanit 2010, 24(Suppl 1):109-113.

45. Whitehead M: Diffusion of ideas on social inequalities in health: a European perspective. Milbank Q 1998, 76(3):469-492. 306.

46. Mackenbach JP: Can we reduce health inequalities? An analysis of the English strategy (1997-2010). J Epidemiol Community Health 2011, 65(7):568-575

47. Brownson RC, Fielding JE, Maylahn CM: Evidence-based public health: a fundamental concept for public health practice. Annu Rev Public Health 2009, 30:175-201.

48. Stronks K: Generating evidence on interventions to reduce inequalities in health: the Dutch case. Scand J Public Health 2002, 59:20-25.

49. Lorenc $T$, Petticrew $M$, Welch $V$, Tugwell P: What types of interventions generate inequalities? Evidence from systematic reviews. J Epidemiol Community Health 2013, 67(2):190-193.

50. House of Commons. Health Committee: Health Inequalities. In Third Report of Session 2008 - 2009. London: The Stationery Office Limited; 2009. http://www. publications.parliament.uk/pa/cm200809/cmselect/cmhealth/286/286.pdf.

doi:10.1186/1471-2458-14-198

Cite this article as: Pons-Vigués et al.: Social and health policies or interventions to tackle health inequalities in European cities: a scoping review. BMC Public Health 2014 14:198.

\section{Submit your next manuscript to BioMed Central and take full advantage of:}

- Convenient online submission

- Thorough peer review

- No space constraints or color figure charges

- Immediate publication on acceptance

- Inclusion in PubMed, CAS, Scopus and Google Scholar

- Research which is freely available for redistribution 